\title{
Reading Development, Word Length and Frequency Effects: An Eye-Tracking Study with Slow and Fast Readers
}

\author{
Sabrina Gerth* and Julia Festman \\ Department of Research and Development in Teacher Education, University College of Teacher Education Tyrol, Innsbruck, \\ Austria
}

OPEN ACCESS

Edited by:

Sascha Schroeder,

University of Göttingen, Germany

Reviewed by:

Ascen Pagan,

Northumbria University,

United Kingdom

Simon Tiffin-Richards, Julius Maximilian University of

Würzburg, Germany

*Correspondence:

Sabrina Gerth

sabrina.gerth@ph-tirol.ac.at

Specialty section:

This article was submitted to

Language Sciences,

a section of the journal

Frontiers in Communication

Received: 17 July 2021 Accepted: 13 September 2021 Published: 28 September 2021

Citation:

Gerth S and Festman J (2021) Reading

Development, Word Length and Frequency Effects: An Eye-Tracking Study with Slow and Fast Readers.

Front. Commun. 6:743113.

doi: 10.3389/fcomm.2021.743113
Research on reading development attempts to explain differences in the reading patterns of adults and children. Previous studies, which typically analyzed word length and frequency effects in developing readers, often focused on dyslexic or dysfluent readers. Similar to previous studies, we investigated the effects of word length and word frequency on the eye movements of children and added several novel aspects: We tested 66 typically developing German-speaking children. Children's oral reading fluency was used as measure of reading ability. Only fast readers $(n=34$, mean age $10.9 \pm$ 0.9 years) and slow readers ( $n=32,11.2 \pm 0.9$ years) participated in an eye-tracking experiment and silently read an age-appropriate original narrative text from a children's book. The analysis of silent reading of the entire text confirmed the earlier group classification. To analyze word length and frequency, we selected 40 nouns as target words in the text. We found significant effects of word length and word frequency for all children in the expected direction. For fast readers, we detected significant interactions of word length and frequency in first fixation duration, gaze duration, and total reading time. These revealed a frequency effect for long, but not short words. This suggests lexical whole-word processing with a fast activation of the word's lexical entry for shorter words and an application of the nonlexical route of the dual route cascaded model (DRC) with a slower lexical access to whole word forms for long words. Slow readers demonstrated a strong sensitivity to word length, indicating a slower or delayed lexical access to orthographic word forms. Additionally, they exhibited weaker word frequency effects. These findings suggest a developmental view of reading in typically developing children in accordance with the DRC, with nonlexical serial decoding as the seemingly prominent reading strategy of slow readers and lexical whole-word recognition as the prominent reading strategy of fast readers.

Keywords: reading, children, eye-tracking, word length effect, word frequency effect, German, dual route cascaded model 


\section{INTRODUCTION}

According to influential models of reading development (e.g., Frith, 1985), children's initial reading acquisition in school begins with decoding. They need to learn the alphabetic code (individual letters) and grapheme-phoneme correspondences (the sound that belongs to each grapheme) to apply them during reading (Grainger and Ziegler, 2011). More recently, approaches to reading acquisition have focused on the recognition of syllables or morphemes rather than letters before beginning readers can transition to whole-word processing (Hasenäcker and Schroeder, 2017). Through reading practice, beginners become familiar with orthographic units (such as words) and link the orthographic structure onto meaning (Shaywitz and Shaywitz, 2005). Only at an advanced level are readers able to decode and comprehend the reading material fluently and effortlessly (e.g., words, sentences, and text).

One model that is commonly used to explain reading processes is the dual-route cascaded model (DRC) of visual word recognition and reading aloud (cf. e.g., Hawelka et al., 2010); it is a computational realization of the dual-route theory of reading (Coltheart et al., 2001). The model postulates two parallel, competitive routes for word recognition. At visual presentation of a word (i.e., a string of letters or graphemes), the model identifies the entire word by sight via the faster lexical route and activates its representation in the orthographic input lexicon. A successful match leads to an activation of the word's semantic and phonological representation to read the word silently or aloud. The activation of a word in the orthographic lexicon is influenced by its frequency of occurrence: High frequency words need less activation and they are recognized faster than low frequency words (Inhoff and Rayner, 1986; Coltheart et al., 2001). Therefore, the lexical route is sensitive to word frequency effects (Yap et al., 2012). The representation of unfamiliar words and nonwords is processed through the model's slower nonlexical route. These words are not yet fully represented in a reader's orthographic input lexicon. Thus, only the graphemephoneme correspondences can be used for decoding. This route is sensitive to word length effects, but not word frequency, because of its serial grapheme-phoneme decoding (Yap et al., 2012).

Developing readers differ from advanced readers in the number of words that are represented in their orthographic lexicon (Coltheart, 2006). At the beginning stages of reading, the novice readers decode words via the nonlexical route using grapheme-phoneme-correspondences. Through regular reading practice, they can quantitatively expand their orthographic lexicon and process words via the lexical route (Coltheart, 2006).

There is empirical evidence for a direct link between lexicon size and reading skills taken from studies with children. A longitudinal study with Dutch children in grades 1-6 found that the children's lexicon size directly relates to their word decoding skills during reading (Verhoeven et al., 2011). In each grade, the authors tested the children's lexicon size and word decoding or reading comprehension. Lexicon size of the younger children (grades 1 and 2) was measured with a receptive vocabulary test during which children saw four pictures and were asked to choose the picture that was uttered aloud by the experimenter (no time limit). To assess their word decoding skills, the younger children were asked to read aloud several words from a word card as accurately and rapidly as possible (time limit of $1 \mathrm{~min}$ per card). The stimulus words were frequently used Dutch words. To assess the older children's (grade 3-6) lexicon size (written vocabulary skills), a multiplechoice item test was administered. Children read a sentence with an underlined target word and then were asked to select the response with the same meaning from four alternatives. One distractor resembled the target word phonologically, another came from the same semantic domain, and the third was previously presented as an oral option during pilot-testing of the task. The older children's reading comprehension was tested with a series of five texts and multiple-choice questions following the reading. These items addressed explicit and implicit meaning relationships between sentences in each of the texts.

The authors found significant developmental progress in children's lexicon size, word decoding, and reading comprehension skills from first to sixth grade (Verhoeven et al., 2011). They also found a strong association between the younger and older children's performance on the vocabulary tests, even though the two tests assessed basic oral vocabulary and the more advanced written vocabulary knowledge. Interestingly, the lexicon size's development of beginning readers (grades 1 and 2) predicted their subsequent reading comprehension. The higher their correctly identified number of lexical items in the receptive vocabulary test, the more successful their word identification and comprehension during reading. This means that the larger the lexicon size, the better the reading skills, since words can be identified, recognized, and mapped more easily onto existing representations in the orthographic lexicon. Apparently, readers with typically developing reading skills benefit from the lexical route more often and identify words by sight as a whole once their orthographic lexicon has been substantially built up.

Contrary to typically developing readers, children with reading difficulties or developmental dyslexia have problems with accurate and fluent processing of larger orthographic units such as words. Therefore, they continue to rely on the word's phonological representation with slower or delayed lexical access (Spinelli et al., 2005; Ziegler and Goswami, 2005; Hawelka et al., 2010). This has been demonstrated in a phonological and orthographic lexical decision task in German with young adults, ages 15-18 (Bergmann and Wimmer, 2008). In the phonological lexical decision task, a dyslexic group and a control group were presented with words (e.g., Taxi), pseudohomophones (e.g., Taksi) that contain a different grapheme realization for the same phoneme, and nonwords (e.g., Tazi). In the phonological lexical decision task, the participants were asked to decide whether the word was pronounced like a real word. In the orthographic lexical decision task, they saw the same words or pseudohomophones and were asked to decide whether the word was spelled correctly. This second task probed the participants' orthographic knowledge on the same items; note that nonwords were judged only in the phonological lexical decision task. The dyslexic group exhibited slower and fewer correct responses compared to the control group for words and pseudohomophones in both tasks. Further, the dyslexic group 
exhibited an increase in error rates and latency from phonological to orthographic decisions, whereas the control group exhibited a decrease of error rates and no difference in latency from phonological to orthographic decisions. Another interesting finding was the distinction between orthographically known and unknown words. Both groups exhibited faster phonological decisions for orthographically known words than for orthographically unknown words and pseudohomophones. The authors interpret this finding as a reliance on the lexical route for orthographically known words in both groups, but the dyslexic group indicated a speed impairment, which was reflected in slower latencies compared to the control group. The authors also revealed that the slower phonological decisions for pseudohomophones and nonwords of the dyslexic group compared to the controls could reflect a speed impairment of the dyslexic group on the nonlexical route (Bergmann and Wimmer, 2008). Therefore, developing readers with difficulties or developmental dyslexia need additional and specific reading practice to improve their decoding skills toward fast whole-word recognition via the lexical route and to build a substantial orthographic lexicon to increase reading fluency (Kuhn and Stahl, 2003).

To directly investigate the movements of the eye during reading, researchers use eye-tracking. This method can especially be employed to shed light on reading processes during silent reading. The different stages of reading development are reflected in characteristic eye movement patterns. Children fixate longer on words and have a higher tendency to fixate on a word more often. They regress more often to previously read words and refixate on words more frequently, probably due to the less targeted fixation on a word that increases the likelihood of a second fixation on the same word. If the eyes land on a word in a position where it cannot be identified, then a refixation on the same word becomes more likely (McConkie et al., 1991). Children also skip fewer words during reading than skilled readers (e.g., adults). This might indicate that the skipped word has already been identified on a prior fixation or without a direct fixation (McConkie et al., 1991). Adults exhibit more developed reading patterns with shorter and more targeted fixations on a word and fewer regressions to previously read material (McConkie et al., 1991; Rayner, 1998).

The E- $Z$ reader model is a computational model of eye movement control, which simulates specific aspects of reading ability (e.g., orthographic knowledge, phonological processing, and sentence comprehension). The E-Z reader model simulates eye-tracking data from adults and children with varying reading levels (Reichle et al., 2013). To disentangle possible influences of linguistic properties on reading behavior, certain parameters of the model can be varied to directly simulate the eye movement patterns. Originally, the model's parameters were established to fit the eye movement patterns of skilled adult readers. These parameters were then systematically changed to simulate the eye movements of children during reading (Mancheva et al., 2015). The respective parameter that required adjustment was the rate of lexical processing. Mancheva et al. suggest "that skilled reading [...] may reflect better orthographic knowledge" (Mancheva et al., 2015, p. 672). These simulations with the E-Z reader model seem to indicate that the differences between children's and adult's eye movements could be explained by the proficiency of lexical processing and orthographic knowledge (Reichle et al., 2013; Mancheva et al., 2015).

Eye-tracking studies with adults have revealed a direct influence of two linguistic properties, word length and frequency, on their eye movements during reading (Rayner, 1998, Rayner, 2009). Generally, compared to short words, long words are fixated longer and more often (Hyönä and Olson, 1995; Just and Carpenter, 1980; Kliegl et al., 2004). High frequency words are read faster and take less time to process than low frequency words (Hyönä and Olson, 1995; Kliegl et al., 2004). Word frequency and word length are highly correlated such that short words are often highly frequent. But even when word frequency remains constant, there is still a word length effect such that long words are fixated for a longer time (Liversedge et al., 2004).

Eye-tracking studies with children also have explored these word property effects-word length and frequency-during oral reading (Hyönä and Olson, 1995; Huestegge et al., 2009; Rau et al., 2014; Rau et al., 2015) and silent reading (Tiffin-Richards and Schroeder, 2015). Children exhibit even stronger word length and frequency effects on eye movement measures than adults (Blythe and Joseph, 2011; Tiffin-Richards and Schroeder, 2015). A few eye-tracking studies with children have included both factors-word length and frequency-in their experimental designs for English (Hyönä and Olson, 1995; Rau et al., 2015), Italian (De Luca et al., 1999), and German (Huestegge et al., 2009; Rau et al., 2014; Rau et al., 2015; Tiffin-Richards and Schroeder, 2015).

We now review the eye-tracking studies with children that investigated word length and frequency effects during reading in English and German. Hyönä and Olson (1995) found robust word length and frequency effects for dyslexic children (12-17 years old) and a control group (9-12 years old) in English oral reading. Gaze durations were longer for long words compared to short words, but this effect stemmed primarily from a greater number of fixations on longer words. Low frequency words were fixated longer than high frequency words in first fixation and gaze duration. Further, the interaction of word length and frequency was significant, with a larger word length effect for low frequency than high frequency words (in first fixation and gaze duration). Rau et al. (2015) found word length effects for two groups of English- and German-speaking children (9-10 years) in gaze duration and word frequency effects in first fixation and gaze duration. Similar to Hyönä and Olson (1995) for English children, they report a stronger word length effect for low frequency compared to high frequency words for the group of German children in first fixation and gaze duration.

In another study, Rau et al. (2014) investigated gaze duration of German-speaking second-graders $(7 ; 10$ years), third-graders ( 8 ; 8 years), fourth-graders $(10 ; 2$ years), and young adults (24; 6 years) for high frequency words, low frequency words, and nonwords of differing length that were embedded in sentences. The authors also found word length effects that were modulated by frequency, but only for skilled readers (third- and fourthgraders and adults). Second-graders exhibited an equal word 
length effect for low frequency words, high frequency words, and nonwords. Short high frequency words were read faster than short low frequency words and short nonwords, suggesting that the former were read by direct lexical access and the latter by serial decoding. In more experienced readers (third- and fourthgraders and adults) the word length effect increased with decreasing word frequency. Third-graders exhibited a continuous reduction in length effects from nonwords to low frequency words through high frequency words. Fourth-graders demonstrated a similar pattern to adults: significant but less marked word length effects for high and low frequency words and strong length effects for nonwords. The authors interpret this finding as the transition from serial nonlexical reading with slower lexical processing (second graders) to direct lexical access of the whole word (third-graders and older). Similarly, in a longitudinal study with second- and fourth-graders in German, Huestegge et al. (2009) found a decreasing word length and frequency effect in gaze duration and total reading time from second grade ( 8 years) to fourth grade (10 years). However, there was no interaction of word length and frequency in any of the eye movement measures or groups.

To our knowledge, there is only one eye-tracking study on silent reading with German-speaking children, conducted by Tiffin-Richards and Schroeder (2015). They measured the eye movements of 75 children (second grade) and an adult control group during silent reading. The authors found a significant word length effect in gaze duration and total reading time and an inversed effect for word length in first fixation duration such that children fixated longer on short words than on long words. However, this could be explained by a higher number of short fixations on long words instead of one single long fixation. Regarding word frequency, the children exhibited significant effects in first fixation duration, gaze duration, and total reading time in the expected direction. The interaction of word length and frequency yielded significant effects in gaze duration and total reading time, with a word length effect that was modulated by frequency: The children's eye movements revealed a greater word length effect for low frequency than high frequency words in gaze duration and total reading time (similar to Hyönä and Olson, 1995; Rau et al., 2014; Rau et al., 2015).

Most of these previously mentioned studies used an oral reading paradigm rather than silent reading. Are reading patterns comparable in both reading modes? To answer this question, we can draw on results from only two eye-tracking studies with adults. Inhoff et al. (2011) compared the two modalities and demonstrated that skilled readers exhibited similar reading patterns during silent and oral reading. In oral reading, adults keep their eyes relatively close to the articulated word with only one or two words as an eye-voice distance. Additionally, Laubrock and Kliegl (2015) report that eye movements are qualitatively similar during silent and oral reading. In oral reading, average fixations were about $50 \mathrm{~ms}$ longer than in silent reading, and saccade lengths were shorter, with fewer regressions due to articulation (Laubrock and Kliegl, 2015). They explain this small lag of the eyes behind the voice with the phonological information that is still held in working memory and is used to comprehend the words. Importantly, Laubrock and Kliegl (2015) found similar word length and frequency effects in both reading modalities (oral and silent). These findings are important because silent reading is easier for children since they do not need to pronounce the words correctly and fluently while still processing and comprehending them. If silent and oral reading yield similar results, then the more natural and easier reading modality can be used for eye-tracking experiments.

\section{Aims of the Present Study}

Often, studies on children's reading behavior compared their eye movements to adults or skilled readers. We extended this research and compared eye movements of two groups of children from the same age group, namely, slow- and fast-reading children categorized according to their oral reading speed. It is still unknown whether or how these groups differ in their reading patterns, since only very few eye-tracking studies have focused on beginning and slightly more advanced readers (i.e., children with 5 to 6 years of reading experience) processing connected texts. Due to the lack of research in this specific area of interest, we aimed to investigate the reading behavior of German-speaking children including several novel aspects. We used text passages taken from real books and collected our data during silent reading in their school environment. These novel aspects were chosen to increase the ecological validity of our data. For that purpose, we used two connected texts from a published children's book as reading material. This approach is different from the use of single words or sentence frames with embedded target words that were often artificially constructed as material in other experimental studies on reading (e.g., Tiffin-Richards and Schroeder, 2015; but see limitations in section Discussion). We opted for this material to investigate the natural reading behavior of embedded target words of our participants in age-appropriate literature. Furthermore, we used a mobile eye-tracking device to be able to collect the data in a familiar environment rather than in a laboratory.

Based on the findings reported in Verhoeven et al. (2011) considering the relatedness of lexicon size and decoding skills in young readers, we assume that, compared to slow readers, fast readers have a larger orthographic lexicon and that they can recognize whole words faster. We predicted that fast and slow readers would differ in speed of word recognition during silent reading of connected text, confirming the group classification based on their speed when reading aloud. Therefore, we expected fast readers to exhibit overall faster word processing on all eyetracking measures, for example, shorter first fixation durations, shorter gaze durations, and a shorter total reading time than slow readers.

Further, we were particularly interested in the effects of word length and frequency in the children's eye movements. We predicted that all children would exhibit word length and frequency effects similar to previous findings in gaze duration and total reading time. Additionally, we had group-specific predictions based on the competitive DRC model. We assumed that lexical processing of slow readers might still rely mainly on the default route of nonlexical decoding. Processes 
based on serial grapheme-phoneme correspondence processes are slower, and, consequently, serial decoding processes should be more strongly influenced by word length than by frequency. Therefore, we expected a stronger word length effect for slow readers than for fast readers. Contrarily, for fast readers, we assumed that processing would not mainly be executed via the nonlexical route. Here, both routes are, indeed, competitive, and the faster lexical route should be used more frequently for processing in this group. Lexical processing involving fast recognition of whole words in fast readers should, therefore, be influenced by lexical characteristics of words such as frequency. Hence, fast readers should exhibit a word length effect that might be modulated by word frequency, and this interaction effect of word length and frequency should be stronger for fast readers than for slow readers. In particular, the word length effect should be stronger for low frequency words compared to high frequency words for fast readers reflected by their more adult-like reading behaviour.

This way, we might detect a developmental distinction between fast lexical and slow nonlexical processing in the group's eye movement patterns, indicating that each group-although having the same age-is likely on a different stage of reading development (following assumptions of the DRC model). More specifically, in the fast-reader group, both routes are in competition and are already used flexibly, depending on the reading material; processing in the other, slower group is still more or less limited to the slow route of nonlexical serial decoding.

\section{MATERIALS AND METHODS}

\section{Participants}

We tested 74 children in fifth grade (27 females, mean age of $10.6 \pm 0.6$ years) and 27 children in sixth grade ( 12 females, mean age of $11.8 \pm 0.8$ years). The children were attending six different primary schools in Brandenburg, Germany.

This study had been approved by the ethics committee of the University of Potsdam and was performed in accordance with the ethical standards laid down in the 1964 Declaration of Helsinki. All children participated voluntarily after their parents provided written and informed consent. The parents received $10 €$ compensation for their children's participation. All participants were German speakers and had normal or corrected-to-normal vision.

\section{Participant Grouping for Preselection Based on Oral Reading Fluency}

A commonly used method to assess oral reading fluency are running records (Fawson et al., 2006). We chose two childfriendly texts; their difficulty was determined by the LIX (Lesbarkeitsindex, an index of readability) as a readability measure that was originally developed by Björnsson in 1968 and adapted for German by Bamberger and Vanecek (1984). The LIX is calculated based on the percentage of long words (more than six letters) and the average sentence length. For fifth- graders, the text comprised 212 words and had a LIX of 32 . For sixth-graders, the text contained 224 words with a LIX of 34. Therefore, the texts were comparable in readability and complexity. Each child was tested individually in a quiet room in their school. At first, the participants read the text silently, then they read the text aloud, and the oral reading was recorded with a dictating machine. Afterward, we calculated words per minute (wpm) that were read correctly. All children read, on average, 111.4 words per minute $(S D=30.9)$. We divided the groups into three subgroups according to their reading speed (slow readers $<100 \mathrm{wpm}$, average readers between 100 and $125 \mathrm{wpm}$, and fast readers $>125 \mathrm{wpm})$.

\section{Eye-Tracking Experiment}

In the eye-tracking study, we recorded the data only for slow and fast readers. We did not collect eye-tracking data for the average readers, because we wanted to have a precise distinction in oral reading fluency between the two groups (i.e., fast and slow readers) at both ends of the reading fluency continuum to determine whether there was a differentiation in reading development in this age group. There was no significant influence of age or grade on the oral reading speed.

Consequently, we recorded eye movement data of 66 children in total; 32 were slow readers (10 females, mean age of $11.2 \pm$ 0.9 years) and 34 were fast readers (25 females, mean age of $10.9 \pm 0.9$ years). The average oral reading speed of the slow readers was $81.7 \mathrm{wpm}(S D=12.9)$ and of the fast readers $139.7 \mathrm{wpm}(S D=9.7)$. An unpaired t-test yielded a significant difference in oral reading speed between the two groups $(t(57.5)=$ $-20.6, p<0.001)$.

\section{Stimulus Texts}

In the eye-tracking experiment, we used two passages from the book "Charlie Bone and the Time Twister" (2003) by Jenny Nimmo. The text passages are age-appropriate and suspenseful but not scary. None of the children had read the texts before participating in the study. To select two comparable narrative texts, we pre-analyzed the passages. The first passage included 89 sentences $(M=13.09$ words per sentence); 1,152 words $(M=$ 10.67 per line); and a LIX of 38 . The second passage contained 87 sentences ( $M=12.44$ words); 1,080 words ( $M=10.50$ per line); and a LIX of 39. Both LIX values indicate low difficulty. The word frequency counts are based on the German childLex corpus (Schroeder et al., 2015) that contains annotated children books directly mirroring the participants' literature input. The two texts did not differ in average word length according to a Welch twosample t-test (text $1: M=5.17, S D=2.6$, range $2-17$ words; text 2: $M=5.20, S D=2.70$, range $2-18$ words; $t(2212.8)=-0.29, p=$ $0.77)$ or word frequency of case-insensitive log10 type per million token (text $1: M=3.47, S D=1.41$; text $2: M=3.45, S D=1.47$; $t(2206.8)=0.30, p=0.77)$.

\section{Word Targets}

Similar to previous studies (Kliegl et al., 1983; Hyönä and Olson, 1995; Tiffin-Richards and Schroeder, 2015), we selected 40 capitalized nouns post-hoc as target words for the word length and frequency analyses. None of these target words were 
positioned at the beginning or end of a sentence to avoid sentence-initiating and wrap-up effects (Tiffin-Richards and Schroeder, 2018). We chose short (three to five letters), long (8-11 letters), high frequency (>2.5 log10 type frequency), and low frequency $(<0.8 \log 10$ type frequency) words. Ten of these were long $(M=9.82, S D=1.08$ letters $)$ and highly frequent $(M=$ $2.76, S D=0.35)$; ten were long $(M=10.00, S D=0.74$ letters $)$ and of low frequency $(M=0.10, S D=0.33)$; ten words were short $(M=4.04, S D=0.81$ letters $)$ and highly frequent $(M=3.62, S D=$ $0.23)$, and ten were short $(M=4.61, S D=0.50$ letters $)$ and of low frequency $(M=0.70, S D=0.84)$. For each of the four categories, we selected five words from one of the two texts ( 40 words from each text). The parameters for word length and frequency were not correlated $(r=0.0521, p=0.6457)$.

\section{Procedure}

To record eye movements during reading, we used a remote eyetracking device (SMI RED 250) with a sampling rate of $250 \mathrm{~Hz}$. The mobile tracking device was attached to a 22 -inch monitor with a screen resolution of $1920 \times 1080$ pixels. The participants sat comfortably at a viewing distance of $58 \mathrm{~cm}$ in front of the monitor. No head support or chin rest was used. The text was presented in Courier New (font size 36) in a black font against a light-gray background using the iViewRED software (Version 4.2.1). The text passages were presented on 10 sequential screens. Each screen contained a maximum of 12 double-spaced lines of text with a maximum of 15 words and 87 characters per line. There were no hyphenated words at line breaks, and we arranged the sentences on the screen such that their endings coincided with line breaks. Each presentation screen was preceded by a fixation cross in the top left-hand corner that triggered the text presentation automatically after $10 \mathrm{~s}$ of cumulative fixations.

Participants were tested individually in a quiet room at their school. At the beginning of the experimental session, participants were orally instructed by the experimenter to read silently for comprehension at a natural pace. Participants were randomly assigned to one of the two text passages. At the beginning of the experimental session, the eye-tracker was calibrated by using nine fixation points on the entire screen. The calibration was repeated until an accuracy of at least $0.5^{\circ}$ was achieved. The eye-tracker was recalibrated after each break and when $x$ - or $y$-axis drifts appeared. Each screen contained a black dot in the lower right corner. We told the participants that, by looking at this dot, they could "turn the page." We had programmed a trigger area around the dot that initiated the presentation of the next screen after $30 \mathrm{~s}$. After the participants finished reading the entire text, we asked them to respond to six comprehension questions on paper. The entire experimental session lasted approximately $40 \mathrm{~min}$.

\section{Data Analysis}

We imported the eye movement data into BeGaze v3.7.40 (SMI) and defined areas of interest (AOIs). Reading and tracking was binocular, but we used only the right eye for data analysis. Each AOI contains one word (including any following punctuation). As eye movement measures, we analyzed first fixation duration (the duration of the very first fixation in an AOI, if any), gaze duration (the sum of
TABLE 1 | Mean first fixation duration, gaze duration and total reading time (in milliseconds) on all words in the texts for slow and fast readers (standard deviations in parentheses).

\begin{tabular}{lcc}
\hline Measure & Slow readers & Fast readers \\
\hline First fixation duration & $268(123)$ & $230(101)$ \\
Gaze duration & $325(161)$ & $266(133)$ \\
Total reading time & $421(203)$ & $335(183)$ \\
\hline
\end{tabular}

all fixations in an AOI until the eye leaves it in any direction, in other words, dwell time) and total reading time (the sum of all fixations in the AOI) (Rayner, 1998). First fixations have been demonstrated to reflect the initial (lexical) processing of a word, whereas gaze duration and total reading time can mirror postlexical word processing (Just et al., 1982). We excluded all fixations on the black dot in the lower-right corner used to proceed to the next screen ( $9.1 \%$ of the data). Additionally, we deleted data with residuals three standard deviations above the participants' mean for each of the three eye movement measures (Baayen and Milin, 2010). Both procedures eliminated in total $12.3 \%$ of all data points.

We used linear-mixed models (lme) to analyze the eye movement data in the $\mathrm{R}$ environment version 3.6.0 ( $\mathrm{R}$ Core Team, 2020) with the lme4 package version 1.1.21 (Bates et al., 2015). We treated participants and AOIs as crossed random effects, and all fixation measures were log-transformed. The factor group (slow or fast reader) was included as a betweensubjects fixed effect, with word length and frequency as withinsubjects fixed effects. Word length and frequency were included as continuous and centered values. The fixation measures were back-transformed from their logarithmic model estimates and are reported in milliseconds. We report regression coefficients relative to the intercept $(b)$, standard errors $(S E)$, and $t$-values for the lme analyses. Only $t$-values larger than $|2.0|$ are considered to be significant at a $p<0.05$ level. Additionally, we report $F$-tests and $p$-values in a results table for ANOVA analyses of the lmemodels.

\section{RESULTS}

\section{Question-Response Accuracy}

The question-response accuracy scores that we obtained from the comprehension questions after the text was read were generally high for all children. The overall mean scores for fast readers were very high (91\%), while slow readers reached an accuracy level of $79 \%$. The difference in accuracy between fast and slow readers was significant $(t=-3.22, p=0.001)$.

\section{Eye Movement Measures for the Entire Text}

We first conducted analyses on all words in the text to generally compare the performance of fast and slow readers during silent reading. Table 1 displays the eye movement measures for both groups on all words in the text.

For the entire text, we found, as expected, significant differences in (silent) reading speed for fast and slow readers 
TABLE 2 | Mean fixation measures (in milliseconds) on the target words for word frequency and length in both groups (standard deviations in parentheses).

\begin{tabular}{lccccc} 
Measure & \multicolumn{2}{c}{ High frequency } & & \multicolumn{2}{c}{ Low frequency } \\
\cline { 2 - 3 } & Short & Long & & Short & Long \\
\hline Fast readers & & & & \\
$\quad$ First fixation duration & $208(85)$ & $202(88)$ & & $224(99)$ & $247(113)$ \\
$\quad$ Gaze duration & $216(90)$ & $264(157)$ & & $245(112)$ & $434(224)$ \\
$\quad$ Total reading time & $250(123)$ & $379(176)$ & & $314(172)$ & $565(205)$ \\
Slow readers & & & & & \\
$\quad$ First fixation duration & $248(110)$ & $249(119)$ & & $262(129)$ & $230(99)$ \\
$\quad$ Gaze duration & $265(124)$ & $389(204)$ & & $299(152)$ & $509(241)$ \\
$\quad$ Total reading time & $322(167)$ & $541(186)$ & & $427(218)$ & $687(188)$
\end{tabular}

in all eye movement measures: Fast readers exhibited shorter first fixation durations $(b=-0.149, S E=0.026, t=-5.670)$, gaze durations $(b=-0.223, S E=0.027, t=-8.338)$, and total reading times $(b=-0.303, S E=0.034, t=-8.814)$ compared to slow readers. This overall faster word processing indicated by all eye-tracking measures confirms the grouping based on (oral) reading speed in the pre-test.

\section{Target Measures}

In the following sections, we present the effects of word length, frequency, and their interaction for fast and slow readers for the selected target words. The dependent eye movement measures on the 40 target words are summarized in Table 2 and are indicated in Figure 1 for each group. Table 3 contains the $F$-tests and $p$-values for all main effects and interactions. Again, we found a significantly faster reading speed for fast compared to slow readers in all eye movement measures on the target words: Fast readers exhibited shorter first fixation durations $(b=-0.133, S E=0.029, t=-4.516)$, gaze durations $(b=-0.198, S E=0.031, t=-6.286)$, and total reading times $(b=-0.299, S E=0.036, t=-8.431)$ than slow readers.

\section{Word Length Effects}

In first fixation duration, we found no significant word length effects for any of the groups. In gaze duration, we revealed a

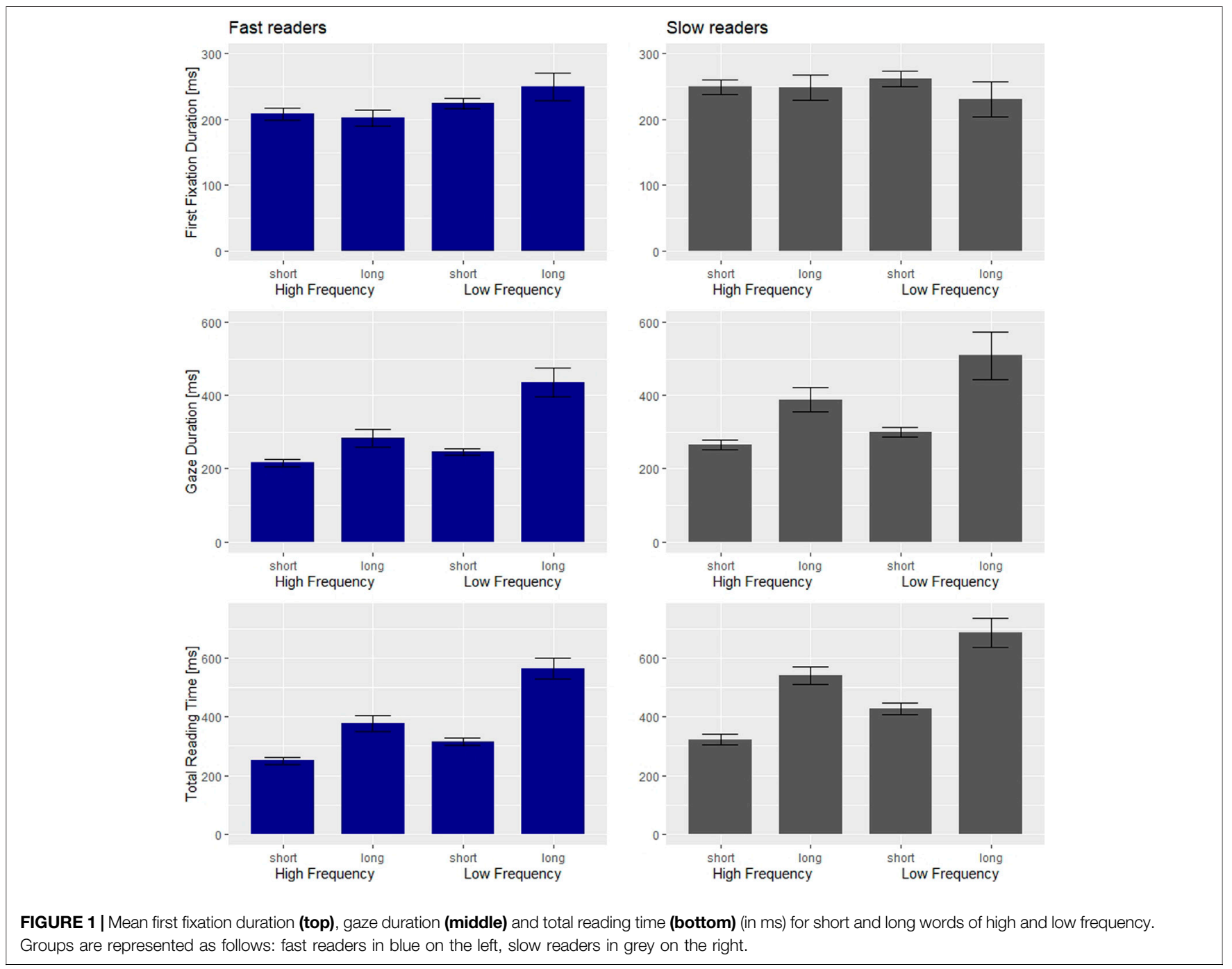


TABLE 3 |F-tests and $p$-values for the main effects and interactions of word length, frequency and group in three eye movement measures (numbers marked in bold indicate significant effects)

\begin{tabular}{|c|c|c|c|c|c|c|}
\hline \multirow[t]{2}{*}{ Effect } & \multicolumn{2}{|c|}{ First fixation } & \multicolumn{2}{|c|}{ Gaze duration } & \multicolumn{2}{|c|}{ Total reading time } \\
\hline & $\boldsymbol{F}$ & $p$ & $\boldsymbol{F}$ & $p$ & $\boldsymbol{F}$ & $p$ \\
\hline Length & 0.05 & 0.823 & 4.25 & 0.039 & 34.60 & $<0.001$ \\
\hline Frequency & 0.63 & 0.426 & 1.88 & 0.170 & 22.68 & $<0.001$ \\
\hline Group & 32.67 & $<0.001$ & 42.69 & $<0.001$ & 75.54 & $<0.001$ \\
\hline Length $\times$ frequency & 0.31 & 0.577 & 5.58 & 0.017 & 4.89 & 0.027 \\
\hline Length $\times$ group & 3.16 & 0.076 & 5.82 & 0.016 & 9.36 & 0.002 \\
\hline Frequency $\times$ group & 1.03 & 0.311 & 0.46 & 0.497 & 0.52 & 0.469 \\
\hline Length $\times$ frequency $\times$ group & 7.05 & 0.008 & 1.54 & 0.215 & 8.26 & 0.004 \\
\hline
\end{tabular}

significant main effect for word length $(b=0.087, S E=0.042, t=$ 2.062), with long words being read $118 \mathrm{~ms}$ longer than short words. In total reading time, we also observed a main effect for word length $(b=0.149, S E=0.025, t=5.882)$, with long words being read $172 \mathrm{~ms}$ longer than short words. Further, we found a significant interaction of word length and group for gaze duration $(b=-0.076, S E=0.032, t=-2.412)$. Fast readers read long words $112 \mathrm{~ms}$ longer than short words. Slow readers read long words $137 \mathrm{~ms}$ longer than short words and exhibited a stronger word length effect in gaze duration. We also found a significant interaction of word length and group for total reading time ( $b=-0.099, S E=0.032, t=-3.059)$. Fast readers read long words $166 \mathrm{~ms}$ longer than short words, whereas slow readers read long words $200 \mathrm{~ms}$ longer than short words and again exhibited a stronger word length effect in total reading time. To summarize, all children revealed the expected word length effect in gaze duration and total reading time. Additionally, the reading behavior of the groups differed in gaze duration and total reading time, with slow readers exhibiting a stronger word length effect compared to fast readers.

\section{Word Frequency Effects}

In first fixation duration and gaze duration, we found no word frequency effect, but in total reading time, we observed a main effect for word frequency $(b=-0.101, S E=0.021, t=-4.762)$. Low frequency words were read $62 \mathrm{~ms}$ longer than high frequency words. In summary, we found a reliable word frequency effect only in one eye movement measure, in total reading time, and no difference in reading behavior between the two groups.

\section{Word Length by Frequency Effects}

In this study, we were mainly interested in the strength of the interaction of the word length and frequency effect in fast- and slow-reading children. We found significant three-way interactions of length, frequency, and group for first fixation duration $(b=-0.050, S E=0.019, t=-2.655)$ and total reading time $(b=-0.061, S E=0.021, t=-2.874)$. We present the results for these two eye-tracking measures separately for the groups, first for fast and then for slow readers.

In first fixation duration for fast readers, we found a significant interaction of word length and frequency $(b=-0.034, S E=0.017$, $t=-2.002)$. We observed a significant word frequency effect for long words $(b=-0.104, S E=0.038, t=-2.773, p=0.013,45 \mathrm{~m})$ but no effect for short words $(b=-0.012, S E=0.019, t=-0.626$, $p=0.541,16 \mathrm{~ms})$. In total reading time, we found significant main effects for length $(b=0.099, S E=0.029, t=3.411, p=0.002)$ and frequency $(b=-0.089, S E=0.023, t=-3.864, p<0.001)$ and $a$ significant interaction of word length and frequency $(b=-0.068$, $S E=0.019, t=-3.761, p<0.001)$. The interaction revealed a greater word length effect for low frequency $(b=0.280, S E=$ 0.037, $t=7.534, p<0.001,251 \mathrm{~ms})$ in comparison to high frequency words $(b=0.167, S E=0.022, t=7.452, p<0.001$, $129 \mathrm{~ms}$ ).

For slow readers, we found less prominent effects. There were no significant interactions of length and frequency, but some main effects for length or frequency. In first fixation duration, we found no significant effects. In total reading time, we found significant main effects for word length $(b=0.120, S E=0.029$, $t=6.838, p<0.001,200 \mathrm{~ms})$ and frequency $(b=-0.114, S E=$ $0.022, t=-5.182, p<0.001,70 \mathrm{~ms})$. Again, we found no interaction of length and frequency, but separate analyses revealed, that the word length effect was greater for low frequency words $(b=0.230, S E=0.040, t=5.691, p<0.001$, $260 \mathrm{~ms})$ than for high frequency words $(b=0.234, S E=0.019, t=$ 12.271, $p<0.001,219 \mathrm{~ms})$.

\section{DISCUSSION}

In this eye-tracking study, we compared eye movements of normally developing fast- and slow-reading children in German with five to six years of reading experience, using a silent reading task of connected text taken from a published children's book and age-appropriate word frequency counts for children. We found that slow readers spend significantly more time on the processing of each word during reading. This has been demonstrated in the analyses of eye movement measures for the entire text (see Table 1) and for target words (see Table 3). More specifically, we investigated the effects of word length and frequency and found significant effects for fast as well as for slow readers. In the separate group analyses, we found a significant interaction of word length and frequency in first fixation duration and total reading time only for the fast readers. For slow readers, we did not find any significant interactions of word length and frequency. We first discuss the results for word length, then word frequency and, afterward, the interaction of both factors.

Regarding word length, our results indicate a consistent effect for fast- and slow-reading children in gaze duration and total 
reading time. Both groups read long words longer than short words. The groups did differ significantly for word length in gaze duration and total reading time (significant interaction of word length and group, see Table 3): Slow readers exhibited a stronger word length effect than fast readers and were apparently more influenced by word length. This suggests that, for slow readers, processing relies more on the nonlexical route than for fast readers, and slow readers' identification of graphemes, syllables, and words might be less efficient. It is likely that slow readers need more time to assemble decoded graphemes and syllables of long words into one word and that processes of lexical access and meaning representation are delayed when compared to the processing speed of fast readers.

Concerning word frequency, we found a main effect in total reading time (see Table 3): Low frequency words received longer fixation durations than high frequency words in both groups. The children revealed this effect only in the late measure of total reading times and not in any of the earlier eye movement measures (first fixation duration or gaze duration). The word frequency effect did not differ between the groups. It appears that word frequency alone cannot explain the different reading patterns of the participants in this study.

The word length factor also interacted with word frequency in the results. Long and low frequency words were processed the longest by all children. Similar to previous findings in eyetracking with children, we replicated a greater word length effect for low frequency words compared to high frequency words (Hyönä and Olson, 1995; Tiffin-Richards and Schroeder, 2015). Hyönä and Olson (1995) found this greater word length effect in the early eye movement measures of first fixation and gaze duration. However, their participants were English-speaking children who read texts aloud while their eyes were being tracked. In this study, we used silent reading of connected texts, so the different reading modality could influence the timing of the effect here. Tiffin-Richards and Schroeder (2015), who used silent reading of German text, found effects quite similar to the ones revealed in our study. Their child participants exhibited longer first fixation durations on short, low frequency words and shorter first fixation durations on long, low frequency words, which are similar to the slow readers in our study. The adult group in their study exhibited the opposite pattern, with the word frequency effect for low frequency words in first fixation duration, being similar to the fast readers in our study. The significant three-way interaction for first fixation duration in our study seems to be driven by this difference in fixation durations for low frequency words: Slow readers fixated longer on short than long low frequency words, while fast readers exhibited the opposite pattern, with longer fixations on long compared to short low frequency words (see Figure 1). All children exhibited similar fixation durations for high frequency words. Tiffin-Richards and Schroeder (2015) suggest that the children's fixation pattern is due to the higher proportion of multiple fixations by children compared to adults. In other words, children often fixate on words more than once. A possible explanation could be a slower lexical access of the slow readers for low frequency words. They attempted to read the short, low frequency words via direct lexical access and, consequently, fixated on these words for a longer time. However, long, low frequency words were too long to be processed at first sight by slow readers, and they needed more refixations to complete lexical processing; or these words were initially processed only superficially and were revisited later for deeper processing. The fast readers in our study revealed quite adult-like reading behavior already in first fixation duration with a word length effect for low frequency words, with results similar to the adults in Tiffin-Richards and Schroeder (2015).

We interpret these results with reference to the DRC (Coltheart et al., 2001). A low frequency word is encountered less often than a high frequency word. This means that either 1) the word is not yet represented in the reader's orthographic lexicon, since it has never been encountered before, or 2) its activation level is very low due to the infrequent occurrence. If, during reading, a word cannot immediately be processed via the fast lexical route and matched onto a mental representation in the orthographic lexicon, word processing is slowed down. In particular, the processing of low frequency words is more likely to fail via a direct lexical matching, especially for young readers who presumably have a smaller vocabulary size than advanced readers and lack the representation of low frequency words. Processing time increases with word length via the nonlexical route, because more graphemes need to be translated into their corresponding phonological representation. If, at this stage, no semantic match can be found to fit the phonological representation, then, as in the choice of 1) above, no entry has been stored and thus no meaning can be directly retrieved. If, however, based on the phonological representation, lexical access to the word form can be realized, this process takes longer, as described in the choice of 2) above, because the activation level of this meaning representation first needs to be raised. Additionally, in languages such as German, long, low frequency words may actually be words that are formed according to the morphological principle of composition. This means that, for example, two nouns are "glued" together to form one new, longer, and less frequent word (e.g., Musik + Genie = Musikgenie, engl. music genius; Mauer + Kronen = Mauerkronen; engl. coping). When reading, it is likely that the two nouns must be recognized individually and their separate meanings must be assembled to represent the full meaning of the long word. These two processes presumably take up more processing time than the lexical access to a single word. Six out of the 10 long, low frequency words were formed by composition, while two of the 10 long but frequent words in our study were based on a different morphological principle: derivation. To identify a derived word during reading, the main carrier of the word's meaning must be isolated and recognized while the affix represents the grammatical meaning of the word (e.g., Dunkelheit $=$ dunkel + -heit, engl. darkness; Sicherheit $=$ sicher + -heit, engl. safety). Other examples for long but frequent words (eight out of 10) were e.g., Großmutter (engl. grandmother), Schatten (engl. shadow), Geschwister (engl. siblings) and Menschen (engl. humans). We suggest that effects of word length in reading, therefore, can be interpreted either as a reliance on the slower nonlexical route necessary due to a small lexicon size and/or difficulties in word segmentation, prolonged processes of lexical access, and additional assembly of meaning.

Interestingly, in gaze duration and total reading time, fast readers exhibited a greater word length effect for low frequency words than 
slow readers did. This might be explained as follows: Fast readers were able to rely on the lexical route to process the high frequency words via direct lexical access (i.e., first fixation duration), but they could not rely on the lexical route for low frequency word (similar to findings with dyslexic readers by Hawelka et al., 2010). In contrast, slow readers revealed a speed-impaired efficiency in reliance on the lexical route for all target words in our study, irrespective of word frequency. This suggests a difference in efficiency of the lexical route for reading from initial reading processes onward. While fast readers demonstrated a better efficiency of available routes and seem to process words more likely via direct lexical access to speed up their reading fluency, slow readers seem to be speed-impaired in their efficiency of the lexical route, similar to dyslexic readers (Hawelka et al., 2010). Furthermore, slow readers seem to have had less time for processing and remembering the content of the text, which was visible in the significant difference of question-word-accuracy between groups.

Due to our choice of method and participants, we encountered some challenges that are relevant to future research. First, we recorded our data with an eye-tracking resolution of only $250 \mathrm{~Hz}$, while the usual choice in reading studies would be $1,000 \mathrm{~Hz}$. We decided to use this particular mobile eye-tracker because we did not want to use a chin rest or head support with our young participants, like some of the cited studies in this paper did (Rau et al., 2014; Rau et al., 2015; TiffinRichards and Schroeder, 2015). Consequently, the child participants could sit relatively still in a chair in a quite natural situation (their school environment) in front of a computer monitor. We acknowledge that this tracking situation came at the cost of a lower tracking resolution. Second, we decided a priori not to track the average readers, since our interest was mainly in shedding light on the reading patterns of two "extreme" reading fluency groups. Thus, we tracked only slow and fast readers based on oral reading fluency in the pre-test. As a reviewer pointed out, we could have collected the data of the average readers and analyzed the reading fluency as a continuous value without dividing the groups for comparison. This would have increased the generalizability of our results. We think that this is absolutely a valid point, and we will strongly consider this in our future research. A third limitation concerns the post-hoc selection of our target words. We chose to present the target words in continuous natural texts taken from a published children's book instead of by constructing sentence frames for each of the words. The sentence frames would have provided a more controlled setting without the opportunity for confounding variables, for example, related to complex morphology. However, we chose this reading material to assess the natural reading of children and were still able to find substantial word length and frequency effects in their eye movements. Children could have encountered these texts in everyday life. We rather wanted to assess the patterns of linguistic word properties, such as word length and frequency of our target words, in an unbiased reading setting rather than in an experimental laboratory situation.

In contrast to earlier eye-tracking studies with children (except Tiffin-Richards and Schroeder, 2015), we used age-appropriate frequency counts. We specifically selected this corpus of German children's books because of spurious results when using word frequency counts from adult corpora in eye-tracking studies with children (Joseph and Liversedge, 2013). Our results reveal interactions of word length and frequency in fifth- and sixthgraders that are similar to those in the study by Tiffin-Richards and Schroeder (2015), who tested second-graders. However, Rau et al., 2014 do not report any interactions in second-graders in an eyetracking study. A possible reason for not finding an interaction in beginning readers could be that they based their frequency counts on an adult corpus rather than on a children's corpus. The discrepancy of results in eye-tracking studies highlights the importance of appropriate reading materials in eye-tracking experiments with children based on suitable frequency counts. Apparently, the word frequency count has a strong impact on beginning readers' eye movement measures and, consequently, on the empirical results. Therefore, the word frequency count, should be based on the reading material that children actually encounter.

Previous eye-tracking research focused mostly on comparing the eye movements of children to skilled adults and interpreted the disparities as stemming from reading development. Studies with child participants have often assessed dyslexic children and agematched control groups to study reading behavior. However, these findings are based on oral reading and not on silent reading, and probably mirror speech production difficulties in children. We need more eye-tracking studies that compare different reading modalities in children to disentangle mixed findings. Future research needs to explore the impact of word length and frequency effects in beginning and slightly more advanced readers. This should be done in relationship to their reading skill, lexicon size, morphological processing skills, and longitudinal developmental differences. Such studies would allow researchers to gain further insights into reading development and the timing of early visual and phonological perception processes, lexical access of word forms and their meanings, and intertwined morphological processes.

\section{DATA AVAILABILITY STATEMENT}

The datasets presented in this article are not readily available because the raw data are property of the University of Potsdam. Requests to access the datasets should be directed to sabrina.gerth@ph-tirol.ac.at.

\section{ETHICS STATEMENT}

The studies involving human participants were reviewed and approved by Ethics committee of the University of Potsdam. Written informed consent to participate in this study was provided by the participants' legal guardian/next of kin.

\section{AUTHOR CONTRIBUTIONS}

Author contributions are as follows: substantial contributions to the conception and design of the project (SG, JF); data acquisition 
and analysis (SG), interpretation of data (SG, JF), drafting of the manuscript (SG) and revision of the manuscript (SG, JF).

\section{FUNDING}

This research was supported by Land Brandenburg, the University of Potsdam, Germany and Land Tirol.

\section{REFERENCES}

Bamberger, R., and Vanecek, E. (1984). Lesen, verstehen, lernen, schreiben: die Schwierigkeitsstufen von Texten in deutscher Sprache. Wien: Jugend und Volk.

Bates, D., Mächler, M., Bolker, B., and Walker, S. (2015). Fitting Linear MixedEffects Models Usinglme4. J. Stat. Soft. 67 (1), 1-48. doi:10.18637/jss.v067.i01

Bergmann, J., and Wimmer, H. (2008). A Dual-Route Perspective on Poor reading in a Regular Orthography: Evidence from Phonological and Orthographic Lexical Decisions. Cogn. Neuropsychol. 25 (5), 653-676. doi:10.1080/ 02643290802221404

Blythe, H. I., and Joseph, H. S. S. L. (2011). "Children's Eye Movements during reading," in Oxford Handbook on Eye Movements (Oxford: Oxford University Press), 643-662. doi:10.1093/oxfordhb/9780199539789.013.0036

Coltheart, M. (2006). Dual Route and Connectionist Models of reading: an Overview. Lond. Rev. Educ. 4 (1), 5-17. doi:10.1080/13603110600574322

Coltheart, M., Rastle, K., Perry, C., Langdon, R., and Ziegler, J. (2001). DRC: A Dual Route Cascaded Model of Visual Word Recognition and Reading Aloud. Psychol. Rev. 108 (1), 204-256. doi:10.1037/0033-295x.108.1.204

De Luca, M., Di Pace, E., Judica, A., Spinelli, D., and Zoccolotti, P. (1999). Eye Movement Patterns in Linguistic and Non-linguistic Tasks in Developmental Surface Dyslexia. Neuropsychologia 37 (12), 1407-1420. doi:10.1016/S00283932(99)00038-X

Fawson, P. C., Ludlow, B. C., Reutzel, D. R., Sudweeks, R., and Smith, J. A. (2006). Examining the Reliability of Running Records: Attaining Generalizable Results. J. Educ. Res. 100 (2), 113-126. doi:10.3200/JOER.100.2.113-126

Frith, U. (1985). Beneath the Surface of Surface Dyslexia. Surface dyslexia: Neuropsychological and Cognitive Studies of Phonological Reading, 301-330.

Grainger, J., and Ziegler, J. C. (2011). A Dual-Route Approach to Orthographic Processing. Front. Psychol. 2, 1-13. doi:10.3389/fpsyg.2011.00054

Harald Baayen, R., and Milin, P. (2010). Analyzing Reaction Times. Int. J. Psychol. Res. 3 (2), 12-28. doi:10.21500/20112084.807

Hasenäcker, J., and Schroeder, S. (2017). Syllables and Morphemes in German reading Development: Evidence from Second Graders, Fourth Graders, and Adults. Appl. Psycholinguist. 38 (3), 733-753. doi:10.1017/S0142716416000412

Hawelka, S., Gagl, B., and Wimmer, H. (2010). A Dual-Route Perspective on Eye Movements of Dyslexic Readers. Cognition 115 (3), 367-379. doi:10.1016/ j.cognition.2009.11.004.A

Huestegge, L., Radach, R., Corbic, D., and Huestegge, S. M. (2009). Oculomotor and Linguistic Determinants of reading Development: A Longitudinal Study. Vis. Res. 49, 2948-2959. doi:10.1016/j.visres.2009.09.012

Hyönä, J., and Olson, R. K. (1995). Eye Fixation Patterns Among Dyslexic and normal Readers: Effects of Word Length and Word Frequency. J. Exp. Psychol. Learn. Mem. Cogn. 21 (6), 1430-1440. doi:10.1037/0278-7393.21.6.1430

Inhoff, A. W., and Rayner, K. (1986). Parafoveal Word Processing during Eye Fixations in reading: Effects of Word Frequency. Percept. Psychophys. 40 (6), 431-439. doi:10.3758/BF03208203

Inhoff, A. W., Solomon, M., Radach, R., and Seymour, B. A. (2011). Temporal Dynamics of the Eye-Voice Span and Eye Movement Control during Oral reading. J. Cogn. Psychol. 23 (5), 543-558. doi:10.1080/20445911.2011.546782

Joseph, H. S. S. L., and Liversedge, S. P. (2013). Children's and Adults' On-Line Processing of Syntactically Ambiguous Sentences during Reading. PLOS ONE 8 (1), e54141. doi:10.1371/journal.pone.0054141

Just, M. A., and Carpenter, P. A. (1980). A Theory of Reading: From Eye Fixations to Comprehension. Psychol. Rev. 87 (4), 329-354. doi:10.1037/0033295x.87.4.329

\section{ACKNOWLEDGMENTS}

We thank the children who participated in our study. Further, we are grateful to Sabine Röttig for providing the data of the oral reading task and helping with participant recruitment of the eyetracking study. We also thank our technical assistant Sebastian Kuhn for help with the collection and pre-processing of the eyetracking data.

Just, M. A., Carpenter, P. A., and Woolley, J. D. (1982). Paradigms and Processes in reading Comprehension. J. Exp. Psychol. Gen. 111 (2), 228-238. doi:10.1037/ 0096-3445.111.2.228

Kliegl, R., Grabner, E., Rolfs, M., and Engbert, R. (2004). Length, Frequency, and Predictability Effects of Words on Eye Movements in reading. Eur. J. Cogn. Psychol. 16 (1-2), 262-284. doi:10.1080/09541440340000213

Kliegl, R., Olson, R. K., and Davidson, B. J. (1983). "On Problems of Unconfounding Perceptual and Language Processes," in Eye Movements in reading: Perceptual and Language Processes. Editor K. Rayner (New York: Academic Press), 333-343. doi:10.1016/b978-0-12-583680-7.50024-2

Kuhn, M. R., and Stahl, S. A. (2003). Fluency: A Review of Developmental and Remedial Practices. J. Educ. Psychol. 95, 3-21. doi:10.1037/0022-0663.95.1.3

Laubrock, J., and Kliegl, R. (2015). The Eye-Voice Span during reading Aloud. Front. Psychol. 6 (1432), 1432. doi:10.3389/fpsyg.2015.01432

Liversedge, S. P., Rayner, K., White, S. J., Vergilino-Perez, D., Findlay, J. M., and Kentridge, R. W. (2004). Eye Movements when reading Disappearing Text: Is There a gap Effect in reading?. Vis. Res. 44 (10), 1013-1024. doi:10.1016/ j.visres.2003.12.002

Mancheva, L., Reichle, E. D., Lemaire, B., Valdois, S., Ecalle, J., and Guérin-Dugué, A. (2015). An Analysis of reading Skill Development Using E-Z Reader. J. Cogn. Psychol. 27 (5), 657-676. doi:10.1080/20445911.2015.1024255

McConkie, G. W., Zola, D., Grimes, J., Kerr, P. W., Bryant, N. R., and Wolff, P. M. (1991). "Children's Eye Movements during reading," in Vision and Visual Dyslexia. Editor J. F. Stein (Boston: CRC Press), 251-262.

R Core Team (2020). R Development Core Team. R: A Language and Environment for Statistical Computing. R Foundation for Statistical Computing. Available at: http://www.r-project.org (Accessed March 31, 2021).

Rau, A. K., Moeller, K., and Landerl, K. (2014). The Transition from Sublexical to Lexical Processing in a Consistent Orthography: An Eye-Tracking Study. Scientific Stud. Reading 18 (3), 224-233. doi:10.1080/ 10888438.2013 .857673

Rau, A. K., Moll, K., Snowling, M. J., and Landerl, K. (2015). Effects of Orthographic Consistency on Eye Movement Behavior: German and English Children and Adults Process the Same Words Differently. J. Exp. Child Psychol. 130, 92-105. doi:10.1016/j.jecp.2014.09.012

Rayner, K. (1998). Eye Movements in reading and Information Processing: 20 Years of Research. Psychol. Bull. 124 (3), 372-422. doi:10.1037/00332909.124.3.372

Rayner, K. (2009). The 35th Sir Frederick Bartlett Lecture: Eye Movements and Attention in reading, Scene Perception, and Visual Search. Q. J. Exp. Psychol. 62 (Issue 8), 1457-1506. doi:10.1080/17470210902816461

Reichle, E. D., Liversedge, S. P., Drieghe, D., Blythe, H. I., Joseph, H. S. S. L., White, S. J., et al. (2013). Using E-Z Reader to Examine the Concurrent Development of Eye-Movement Control and reading Skill. Develop. Rev. 33 (2), 110-149. doi:10.1016/j.dr.2013.03.001

Schroeder, S., Würzner, K.-M., Heister, J., Geyken, A., and Kliegl, R. (2015). childLex-Eine lexikalische Datenbank zur Schriftsprache für Kinder im Deutschen. Psychologische Rundschau 66 (3), 155-165. doi:10.1026/00333042/a000275

Shaywitz, S. E., and Shaywitz, B. A. (2005). Dyslexia (Specific reading Disability). Biol. Psychiatry 57 (11), 1301-1309. doi:10.1016/ j.biopsych.2005.01.043

Spinelli, D., De Luca, M., Di Filippo, G., Mancini, M., Martelli, M., and Zoccolotti, P. (2005). Length Effect in Word Naming in Reading: Role of Reading Experience and Reading Deficit in Italian Readers. Develop. Neuropsychol. 27 (2), 217-235. doi:10.1207/s15326942dn2702_2 
Tiffin-Richards, S. P., and Schroeder, S. (2018). The Development of WrapUp Processes in Text reading: A Study of Children's Eye Movements. J. Exp. Psychol. Learn. Mem. Cogn. 44 (7), 1051-1063. doi:10.1037/ xlm0000506

Tiffin-Richards, S. P., and Schroeder, S. (2015). Word Length and Frequency Effects on Children's Eye Movements during Silent reading. Vis. Res. 113 (PA), 33-43. doi:10.1016/j.visres.2015.05.008

Verhoeven, L., van Leeuwe, J., and Vermeer, A. (2011). Vocabulary Growth and reading Development across the Elementary School Years. Scientific Stud. Reading 15 (1), 8-25. doi:10.1080/10888438.2011.536125

Yap, M. J., Balota, D. A., Sibley, D. E., and Ratcliff, R. (2012). Individual Differences in Visual Word Recognition: Insights from the English Lexicon Project. J. Exp. Psychol. Hum. Percept. Perform. 38 (1), 53-79. doi:10.1037/a0024177

Ziegler, J. C., and Goswami, U. (2005). Reading Acquisition, Developmental Dyslexia, and Skilled Reading across Languages: A Psycholinguistic Grain Size Theory. Psychol. Bull. 131 (1), 3-29. doi:10.1037/0033-2909.131.1.3
Conflict of Interest: The authors declare that the research was conducted in the absence of any commercial or financial relationships that could be construed as a potential conflict of interest.

Publisher's Note: All claims expressed in this article are solely those of the authors and do not necessarily represent those of their affiliated organizations, or those of the publisher, the editors and the reviewers. Any product that may be evaluated in this article, or claim that may be made by its manufacturer, is not guaranteed or endorsed by the publisher.

Copyright $\odot 2021$ Gerth and Festman. This is an open-access article distributed under the terms of the Creative Commons Attribution License (CC BY). The use, distribution or reproduction in other forums is permitted, provided the original author(s) and the copyright owner(s) are credited and that the original publication in this journal is cited, in accordance with accepted academic practice. No use, distribution or reproduction is permitted which does not comply with these terms. 